BMJ Open Sport \& Exercise Medicine

\title{
Normal platelet function in platelet concentrates requires non-platelet cells: a comparative in vitro evaluation of leucocyte-rich (type 1a) and leucocyte- poor (type $3 b$ ) platelet concentrates
}

\author{
William R Parrish, ${ }^{1}$ Breana Roides, ${ }^{1}$ Julia Hwang, ${ }^{1}$ Michael Mafilios, ${ }^{2}$ \\ Brooks Story, ${ }^{1}$ Samir Bhattacharyya ${ }^{1}$
}

\begin{abstract}
Background: Therapeutic success of platelet-rich plasma (PRP) may vary based on the composition and preparation method. The objective of this study was to evaluate the cellular components of platelet concentrates produced by a leucocyte-rich (LR-PRP) and a leucocyte-poor PRP systems (LP-PRP). Methods: Parameters evaluated included platelet recovery, platelet concentration, red blood cell (RBC) and white blood cell (WBC) composition, platelet growth factor release and stimulation of human tendon cell proliferation in vitro.
\end{abstract}

Results: Platelet recoveries were $52 \%$ for LP-PRP and $89 \%$ for LR-PRP. LR-PRP demonstrated greater reproducibility with a $4.2 \%$ coefficient of variation (CV) compared with $19.4 \%$ for LP-PRP $(p<0.001)$. LR-PRP demonstrated a greater increase in platelet concentration (7.9-fold) than LP-PRP (2.2-fold; $p<0.001)$. LP-PRP showed 5.0-fold reductions in WBCs, while LR-PRP showed a 4.0-fold increase $(p<0.001)$. LP-PRP reduced RBCs to a haematocrit of 0.25 , while LR-PRP reduced haematocrit to 11.8 . LP-PRP did not coagulate robustly on reactivation with $\mathrm{CaCl}_{2}$, and released significantly lower levels of epidermal growth factor (EGF) and transforming growth factor $\beta 1$ (TGF- $\beta 1$ ) than whole blood $(p<0.03)$. LP-PRP also did not stimulate tendon cell proliferation greater than whole blood. In contrast, LR-PRP showed increases in each growth factor on activation with $\mathrm{CaCl}_{2}(p<0.01)$ and stimulated greater proliferation $(p<0.05)$ compared with whole blood. Forced activation of LP-PRP with exogenous thrombin rescued the coagulation deficiency and induced greater growth factor release than comparable whole blood $(p<0.03)$.

Conclusions: These data suggest that non-platelet cellular components in platelet concentrates are important for proper platelet function, including thrombin generation, growth factor release and clot retraction.

\section{INTRODUCTION}

Platelet-rich plasma (PRP) is an autologous, biologically active derivative of whole blood

\section{What are the new findings?}

- Compared with whole blood, leucocyte-poor platelet-rich plasma (PRP) contains $<1 \%$ and $20 \%$ of red blood cells and white blood cells, respectively, and it is deficient in coagulation, clot retraction and platelet growth factor release when reactivated with $\mathrm{CaCl}_{2}$.

- Platelet releasate (serum) from reactivated leucocyte-poor PRP containing greater than twofold increased platelet concentration does not stimulate greater tendon cell proliferation than whole blood serum in vitro.

- Exogenous thrombin addition rescues coagulation and clot retraction deficits and significantly improves platelet growth factor release in leucocyte-poor PRP.

- This study demonstrates that non-platelet cellular components in platelet concentrates are important for proper platelet function, including thrombin generation, clot retraction and growth factor release.

that contains therapeutic platelet concentrations elevated at least fivefold above baseline. ${ }^{1}$ By concentrating platelets into PRP, a large reservoir of autologous growth factors is generated, which if administered appropriately can establish an optimal environment for accelerated healing, including the reduction of pain and resolution of inflammation. $^{2-7}$ There are currently more than 2 dozen commercially available systems in the worldwide market that concentrate whole blood into a platelet-rich substance. These various devices and techniques have resulted in a wide range of PRP final products that can vary greatly, especially in the concentration of platelets and the inclusion or exclusion of leucocytes and red blood cells 
(RBCs). A current area of debate is how non-platelet components of whole blood contribute to the biological activity of PRP. ${ }^{8-14}$ In particular, some authors have suggested based on preclinical studies that RBCs and white blood cells (WBCs) may be detrimental as these cells may participate in inflammatory reactions that can lead to damage in the treated tissue. ${ }^{14-16}$ Although these concerns remain unsubstantiated in the clinical literature, ${ }^{9} 13$ an important question that does remain is whether nonplatelet cellular components of PRP contribute to its biological activity in a manner that may impact platelet activation and growth factor release, and thus the therapeutic utility of the PRP.

In the context of whole blood, the intimate and dynamic interactions between platelets and other cells is crucial for providing the physiological mechanisms that prevent infection and promote healing in the event of an injury. For example, collagen-activated platelets are known to stimulate RBCs to activate robust prothrombin conversion. ${ }^{12}$ 17-20 This RBC-dependent amplification mechanism generates sufficient thrombin to convert the forming platelet plug into a fibrin clot and ensures appropriate levels of reciprocal platelet activation and growth factor release that are proportional to the injury. ${ }^{20}$ Equally important are interactions between platelets and leucocytes that coordinate the inflammatory and immune responses to match the antimicrobial and repair needs of the damaged tissue. ${ }^{11}{ }^{17} \quad 20-28$ These coordinated activities control the level of inflammation caused by platelet activation and ultimately trigger the healing cascade, which results in the restoration of the damaged tissue to a healthy state. ${ }^{2} 45729$ Therefore, similar intercellular platelet interactions that are foundational in normal wound repair might also function to influence the bioactivity of platelets in PRP. To investigate this hypothesis, we evaluated two commercially available PRP preparation systems, the Arthrex Autologous Conditioned Plasma (ACP) dual syringe system and the Mitek Sports Medicine PEAK PRP system, that generate platelet concentrates on each end of the spectrum of PRP (table 1). ${ }^{5}$

ACP is leucocyte poor (LP) and RBC depleted (a type 3b PRP), whereas PEAK is leucocyte rich (LR) and contains RBCs (a type 1a PRP). ${ }^{5}$ The objectives of this study were (1) to evaluate the cellular components of platelet concentrates produced by a LR-PRP system and a LP-PRP system, (2) to assess platelet growth factor

\begin{tabular}{lll} 
Table 1 & Platelet-rich plasma classification system & \\
\hline Type & White blood cells & Activated? \\
\hline 1 & Increased over baseline & No \\
2 & Increased over baseline & Yes \\
3 & Minimal or no white blood cells & No \\
4 & Minimal or no white blood cells & Yes \\
\hline A: Platelets $>5 \times$ baseline. & \\
B: Platelets $<5 \times$ baseline. &
\end{tabular}

release following reactivation of those platelet concentrates with $\mathrm{CaCl}_{2}$, and (3) to evaluate the ability of those platelet concentrates to stimulate proliferation of tendon cells in vitro. Comparative evaluation studies of this nature are important for the advancement of the PRP field because evidence in the literature suggests that therapeutic success of PRP may vary based on the preparation method and PRP composition. ${ }^{5} 1013$ Thus, there is a clear need to identify components that contribute to an ideal formulation that optimises the biological potential of platelet concentrates, including the reliability of the device to generate a reproducible platelet concentrate.

\section{METHODS}

\section{Study design}

This study evaluates the cellular characteristics and reproducibility of platelet concentrates generated by an LR-PRP system (type 1a) and a LP-PRP system (type 3b). Parameters evaluated were platelet recovery, platelet concentration, RBC and WBC composition, platelet growth factor release following reactivation with $\mathrm{CaCl}_{2}$ and the capacity of the releasate to stimulate human tendon cell proliferation in vitro.

\section{Generation of platelet concentrates and haematology measurements}

Whole fresh human blood anticoagulated with $12 \%$ anticoagulant citrate dextrose solution A (ACD-A) was obtained from Research Blood Components, LLC (Brighton, Massachusetts, USA). Platelet concentrates from the LR-PRP and LP-PRP systems were prepared in parallel for each donor according to the Instructions for Use contained in the device packaging. PRP was collected immediately following preparation and an aliquot for haematology analysis was diluted 1:3 with sterile phosphate buffered saline (PBS) containing $10 \mathrm{mM}$ EDTA to ensure that platelet counts would fall within the calibrated linear range of detection (40 000-700 000 platelets $/ \mu \mathrm{L}$ ) for the Horiba ABX Micros 60 haematology analyser. Each sample was read three times and the mean haematology values were reported for each donor.

\section{Serum generation}

A total of $3 \mathrm{~mL}$ of ACD-A anticoagulated whole blood, LP-PRP, LR-PRP or platelet-poor plasma (PPP) was measured by graduated pipette and placed into sterile polypropylene tubes. Samples were reactivated physiologically with a final concentration of $25 \mathrm{mM}$ calcium chloride to counteract the ACD-A anticoagulant for $80 \mathrm{~min}$ at room temperature. Serum samples were then prepared by centrifugation at $1000 \mathrm{~g}$ for $10 \mathrm{~min}$ to pellet fibrin clots, and the fluid portion (serum) was collected into fresh sterile polypropylene tubes. The serum samples were centrifuged again at $1000 \mathrm{~g}$ for $10 \mathrm{~min}$ to remove residual cells and debris. The cleared serum was aliquoted into Nalgene cryovials and frozen at $-80^{\circ} \mathrm{C}$ 
until assayed for growth factor content and the capacity to stimulate tendon cell proliferation in vitro. For forced PRP activation, 100 units of bovine thrombin were

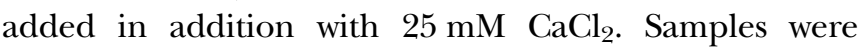
immediately inverted several times to ensure mixing, and were incubated at room temperature for $60 \mathrm{~min}$ to allow complete coagulation. Serum samples were prepared as described above. No serum aliquots were freeze-thawed more than once prior to use in any assay.

\section{Tendon cell proliferation studies}

Normal healthy primary human patellar tendon fibroblasts (tenocytes) were purchased from Articular Engineering, LLC (Northbrook, Illinois) at passage \#1. Cultures were expanded to passage \#6 to ensure homogenous cell populations and appropriate acclimation to in vitro culture conditions. Cells were maintained at $37^{\circ}$ $\mathrm{C}$ under $5 \% \mathrm{CO}_{2}$ in Dulbecco's-Modified Eagle Medium-F12 (DMEM-F12) supplemented with 10\% fetal bovine serum (FBS), 1 xantibiotic/antimycotic and $2 \mathrm{mM}$ L-glutamine. For studies, cells were seeded into tissue culture-treated black-walled clear bottom 96-well plates at $1 \times 10^{3}$ cells $/$ well in $100 \mu \mathrm{L}$ volume. Cultures were washed once with $100 \mu \mathrm{L}$ of DMEM-F12 $24 \mathrm{~h}$ after seeding and synchronised for $24 \mathrm{~h}$ by serum restriction to $1 \%$ FBS. Eight wells per condition were treated in parallel (quadruplicate wells on duplicate plates) with serum derived from whole blood (positive control), PPP (negative control) or platelet concentrates from LP-PRP or LR-PRP. All serum dilutions were prepared in DMEM-F12 supplemented with 1×antibiotic/antimycotic, $2 \mathrm{mM}$ L-glutamine and $0.25 \%$ FBS to maintain viability of the $0 \%$ serum-treated cultures. Cultures were incubated at $37^{\circ} \mathrm{C}$ under $5 \% \mathrm{CO}_{2}$ for 4 days. Wells were washed 1×with PBS to remove residual media, and relative proliferation was determined by CellTiter-Glo assay using a Molecular Diagnostics Spectramax M5 plate reader to measure luminescence.

\section{Growth factor measurements}

ELISA assay kits (Quantikine, R\&D systems) were used to analyse the serum levels of five representative platelet growth factors (epidermal growth factor (EGF), transforming growth factor $\beta 1$ (TGF- $\beta 1$ ), platelet-derived growth factor $\mathrm{AB}$ (PDGF-AB), vascular endothelial growth factor (VEGF) and stromal-derived factor $1 \alpha$

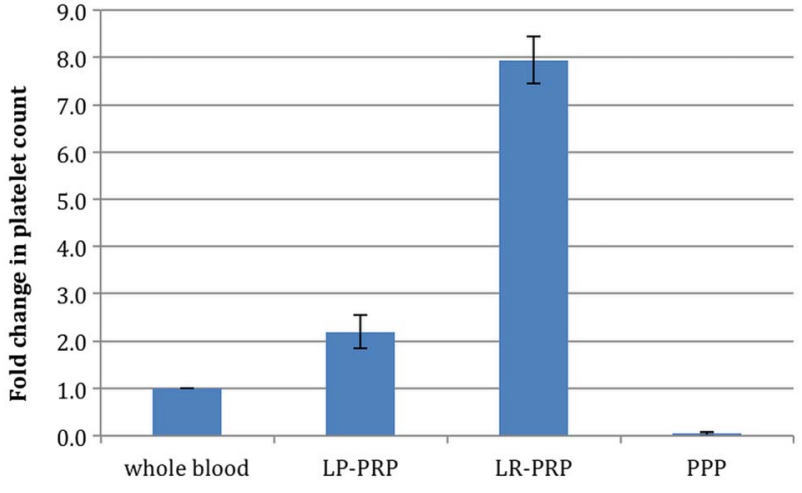

Figure 1 Fold change in platelet concentration. Bars represent the mean $\pm S D$ of the fold increase in platelet content $(n=12$ donors). Fold increase=platelet count in platelet concentrate/whole blood platelet count.

$(\mathrm{SDF}-1 \alpha))$. PPP that was prepared in parallel for each donor served as a negative control. Samples were assayed in duplicate and assay results were considered valid when $r^{2}$ values for the standard curves were greater than 0.99 and sample detection was within the linear range of the standard curve. Samples not within the linear detection range were diluted accordingly and reassayed.

\section{Statistical analyses}

Comparisons between groups were conducted using paired one-tailed Student t test, or by one-way analysis of variance (ANOVA) where indicated. Differences were considered significant when $\mathrm{p}<0.05$. The coefficient of variation, a ratio of the $\mathrm{SD}$ to the mean expressed as a per cent $(\% \mathrm{CV}=(\mathrm{SD} /$ mean $) \times 100)$, was also assessed for platelet recovery where a lower coefficient of variation (CV) indicates less variability.

\section{RESULTS}

\section{Platelet composition}

The mean whole blood platelet count was $224 \times 10^{3} / \mu \mathrm{L}$ (table 2). Each system significantly increased the platelet count compared with baseline levels $(p<0.001)$. LR-PRP demonstrated significantly greater increases in platelet concentration over baseline (7.9-fold) than LP-PRP (2.2-fold increase; $\mathrm{p}<0.001$ ) (figure 1 and table 2). In addition, LP-PRP contained just over $50 \%$ of the total

Table 2 Platelet parameters

\begin{tabular}{lcllll}
\hline & $\begin{array}{l}\text { Mean platelet count } \\
\left(\times \mathbf{1 0}^{3} / \boldsymbol{\mu L}\right)\end{array}$ & Per cent recovery & Fold increase & $\begin{array}{l}\text { Coefficient of } \\
\text { variation (\%) }\end{array}$ & p Value \\
\hline Whole blood & 224.2 & NA & 1.0 & NA & $<0.001$ \\
LP-PRP & 485.9 & 52 & 2.2 & 19.4 & $<0.001$ \\
LR-PRP & 1772.7 & 89 & 7.9 & 4.2 & NA \\
PPP & 8.6 & NA & 0.0 & NA & NA \\
\hline
\end{tabular}

LP-PRP, leucocyte-poor platelet-rich plasma system; LR-PRP, leucocyte-rich platelet-rich plasma system; NA, not applicable; PPP, platelet-poor plasma. 


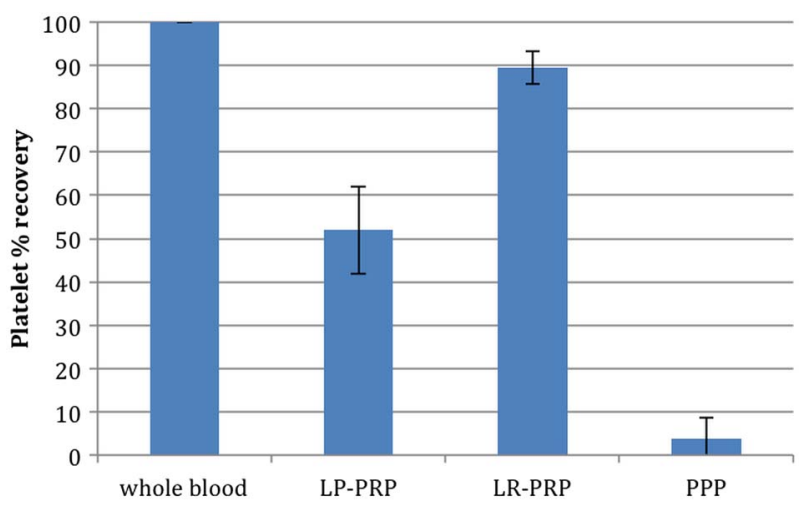

Figure 2 Platelet recovery. Bars represent the mean \pm SD of the percentage of total platelets recovered in platelet concentrates ( $n=12$ donors). Per cent platelet recovery=(total platelet count of platelet concentrate (concentration $\times$ total volume)/total platelet count of whole blood (concentration $\times$ total volume) $) \times 100$.

available platelets, while LR-PRP contained almost $90 \%$ (figure 2 and table 2). LR-PRP demonstrated a greater reproducibility in platelet recovery with a $4.2 \%$ CV compared with a $19.4 \% \mathrm{CV}$ for LP-PRP $(\mathrm{p}<0.001)$ (table 2$)$.

\section{RBC composition}

The mean whole blood baseline RBC count was $4.04 \times 10^{6} / \mu \mathrm{L}$, resulting in a mean haematocrit of 32.9 (table 3). Each of the systems reduced RBCs compared with whole blood. Haematocrit levels for LP-PRP and LR-PRP were 0.25 and 11.8 , respectively $(\mathrm{p}<0.01)$ (table 3).

\section{WBC composition}

The mean whole blood WBC count was $4.9 \times 10^{3} / \mu \mathrm{L}$ (table 4). WBCs were reduced by fivefold in LP-PRP $(\mathrm{p}<0.001)$, whereas LR-PRP showed a 4.0-fold increase in WBCs $(\mathrm{p}<0.001)$ (table 4). LP-PRP contained just below $5 \%$ of the total available WBCs, while LR-PRP contained approximately $45 \%$ (table 4 ).

\section{Tendon cell proliferation}

Human tendon fibroblast cells were grown in vitro and exposed to varying concentrations of serum derived from either whole blood, PPP or platelet concentrates generated by LP-PRP or LR-PRP. Untreated cultures gave a very consistent baseline measurement with an across group mean of $209 \pm 5$ luminescence units, demonstrating excellent interassay and intra-assay repeatability and a common assay start point with respect to cell number per well ( $p>0.98$ by one-way ANOVA) (table 5 ). PPP stimulated a linear concentration-dependent proliferative response in human tenocytes $\left(r^{2}=1\right)$ (table 5). Thus, to specifically compare the effects of platelet-derived growth factors on tendon cell proliferation, cell responses to serum were normalised to the PPP response and expressed as per cent (\%) stimulation over PPP (figure 3).

Serum from whole blood, LP-PRP and LR-PRP each stimulated a concentration-dependent proliferative response from tendon cells (figure 3). In fact, although the LP-PRP system preparations contained a 2.2-fold increase in platelets over whole blood (figure 1), the serum derived from those platelets did not evoke an enhanced proliferation response compared with whole blood ( $p>0.7$ by one-way ANOVA; figure 3 ). These results strongly suggest that the LP-PRP system preparations are deficient in platelet activation and growth factor release. In contrast, LR-PRP stimulated a proliferation dose-response that was significantly greater than that of either LP-PRP or whole blood ( $p<0.05$ for each by one-way ANOVA; figure 3 and table 5). More specifically, the serum from LR-PRP at a concentration of $10 \%$ (volume:volume) in the culture medium stimulated robust proliferation that was sufficient to cause the cultures to grow to confluence, saturating the assay system. This finding is important because normal somatic cells such as the tendon fibroblasts used in these studies exhibit contact-dependent inhibition of proliferation when grown in culture, whereby cells that are surrounded by other cells can no longer respond to serum growth signals. ${ }^{30}$ This effect is evidenced by the apparent flattening of the slope of the serum response curve between $5 \%$ and $10 \%$ serum for LR-PRP (figure 3).

\section{Growth factor analysis}

Whole blood and LR-PRP achieved a robust activation of the coagulation cascade following reactivation with $\mathrm{CaCl}_{2}$, including strong clot retraction. In contrast, LP-PRP and PPP displayed donor-dependent irregularity in the robustness and degree of clotting, and none of the LP-PRP or PPP clots demonstrated retraction to the

Table 3 Red blood cell parameters

\begin{tabular}{lllllc}
\hline & $\begin{array}{l}\text { Mean RBC count } \\
(\times \mathbf{1 0} / \boldsymbol{\mu L})\end{array}$ & Per cent depletion & $\begin{array}{l}\text { Coefficient of } \\
\text { variation }(\%)\end{array}$ & Fold change & Haematocrit \\
\hline Whole blood & 4.04 & NA & NA & 1.00 & 32.9 \\
LP-PRP & 0.05 & 99.8 & 0.31 & 0.01 & 0.25 \\
LR-PRP & 1.50 & 96.8 & 1.04 & 0.37 & 11.8 \\
PPP & 0.00 & 100.0 & NA & NA & 0 \\
\hline
\end{tabular}

LP-PRP, leucocyte-poor platelet-rich plasma system; LR-PRP, leucocyte-rich platelet-rich plasma system; NA, not applicable; PPP, platelet-poor plasma; RBC, red blood cell. 
Table 4 White blood cell parameters

\begin{tabular}{lclllll}
\hline & $\begin{array}{l}\text { Mean WBC } \\
\text { count } \\
\left(\times \mathbf{1 0}^{\mathbf{3}} / \boldsymbol{\mu L}\right)\end{array}$ & $\begin{array}{l}\text { Per cent } \\
\text { recovery }\end{array}$ & $\begin{array}{l}\text { Mean fold } \\
\text { increase }\end{array}$ & $\begin{array}{l}\text { Coefficient of } \\
\text { variation }(\%)\end{array}$ & $\begin{array}{l}\text { Mean platelet: } \\
\text { WBC ratio }\end{array}$ & $\begin{array}{l}\text { p Value (2-tailed paired } \\
\text { t test) vs LR-PRP system }\end{array}$ \\
\hline $\begin{array}{l}\text { Whole } \\
\text { blood }\end{array}$ & 4.9 & 100.0 & 1.0 & NA & 46 & $<0.001$ \\
LP-PRP & 1.0 & 4.1 & 0.2 & 100 & 486 & $<0.001$ \\
LR-PRP & 19.3 & 44.9 & 4.0 & 26 & 92 & NA \\
PPP & 0.0 & 0.0 & NA & NA & NA & NA
\end{tabular}

LP-PRP, leucocyte-poor platelet-rich plasma system; LR-PRP, leucocyte-rich platelet-rich plasma system; NA, not applicable; PPP, platelet-poor plasma; WBC, white blood cells.

extent of whole blood (data not shown). This indicates a deficiency in activation of the coagulation cascade and also in platelet function, since clot retraction is a platelet-driven process. ${ }^{31}$ Further consistent with a platelet functional deficiency in LP-PRP, a lower level of growth factors compared with whole blood serum was found despite that LP-PRP contained 2.2-fold more platelets (figures 1 and 4 and table 6 ). In fact, the levels of EGF and TGF- $\beta 1$ were significantly lower in the LP-PRP serum compared with whole blood $(p<0.03$ for both; figure 4 and table 6 ). In contrast, LR-PRP demonstrated significantly higher growth factor levels compared with serum from whole blood $(\mathrm{p}<0.01$ for each; figure 4 and table 6).

The failure of LP-PRP to demonstrate robust coagulation and platelet growth factor release in response to reactivation with $\mathrm{CaCl}_{2}$ suggested that LP-PRP may be deficient in thrombin generation under physiological conditions. To test this, LP-PRP was directly activated with 100 units of bovine thrombin in addition to $25 \mathrm{mM}$ $\mathrm{CaCl}_{2}$, and growth factor measurements were conducted on the serum samples. Robust coagulation was induced in each sample within seconds of the addition of thrombin, indicating that exogenous thrombin can indeed rescue the clotting deficiency observed for LP-PRP. Forced activation with exogenous thrombin also improved platelet growth factor release in LP-PRP compared with activation with $\mathrm{CaCl}_{2}$ alone, respective to whole blood controls (figure 5). Taken together, these data indicate that LP-PRP preparations are deficient in thrombin generation on reactivation with $\mathrm{CaCl}_{2}$.

\section{DISCUSSION}

Evidence in the literature suggests that clinical success of PRPs may vary based on the preparation method and
PRP composition. ${ }^{9} 1332$ Thus, there is a need to evaluate PRP formulations to discern the role of non-platelet cellular components in determining the potential efficacy of PRP generated by different systems. The primary objectives of the current study were to characterise the cellular components of LR-PRP (a type 1a PRP containing RBCs) and LP-PRP (a type 3b PRP, ie, RBC depleted) and to examine the contributions that RBCs and WBCs collectively have on platelet growth factor release and the efficacy of the releasate to stimulate tendon cell proliferation in vitro. LR-PRP contained almost $90 \%$ of the available platelets, resulting in approximately 7.9-fold increase over whole blood with a $4.2 \%$ coefficient of variation, which indicates a high degree of reproducibility in platelet concentration. WBCs were concentrated approximately four-fold over whole blood in LR-PRP, which was sufficient to maintain platelet to $\mathrm{WBC}$ ratios within a physiological range for each donor tested. The platelet to WBC ratio is a metric that has recently gained attention as a parameter that may be important for the anabolic activity of PRP, ${ }^{14} 33$ although the influence of this ratio on PRP bioactivity remains controversial. LR-PRP RBC levels were reduced to a haematocrit of 11.8 , almost threefold lower than whole blood. The LR-PRP was type 1A, and demonstrated robust physiological responsiveness to reactivation with $\mathrm{CaCl}_{2}$. Complete coagulation with robust clot retraction was seen with LR-PRP, and this was evidenced by high serum growth factor content (table 6 and figure 4) and strong stimulation of tendon cell proliferation in vitro (table 5 and figure 3). Indeed, tendon cells stimulated with $10 \%$ serum from LR-PRP saturated the growth surface of the cell culture dishes, thus reaching the plateau for proliferation in vitro. Overall, these results indicate excellent platelet viability in the LR-PRP and suggest that robust platelet activity should be expected in a physiological setting.

Table 5 Tenocyte proliferation measurement (light units \pm SEM)

\begin{tabular}{llllll}
\hline Serum concentration & $\mathbf{0} \%$ & $\mathbf{1 \%}$ & $\mathbf{2 . 5 \%}$ & $\mathbf{5 \%}$ & $\mathbf{1 0 \%}$ \\
\hline Whole blood & $208(29)$ & $271(19)$ & $515(46)$ & $1087(139)$ & $2622(95)$ \\
LP-PRP & $214(19)$ & $281(19)$ & $554(62)$ & $1240(134)$ & $2656(92)$ \\
LR-PRP & $203(17)$ & $384(46)$ & $1001(91)$ & $2517(180)$ & $4372(481)$ \\
PPP & $210(16)$ & $269(10)$ & $370(11)$ & $530(23)$ & $795(58)$ \\
\hline LP-PRP, leucocyte-poor platelet-rich plasma system; LR-PRP, leucocyte-rich platelet-rich plasma system; PPP, platelet-poor plasma.
\end{tabular}




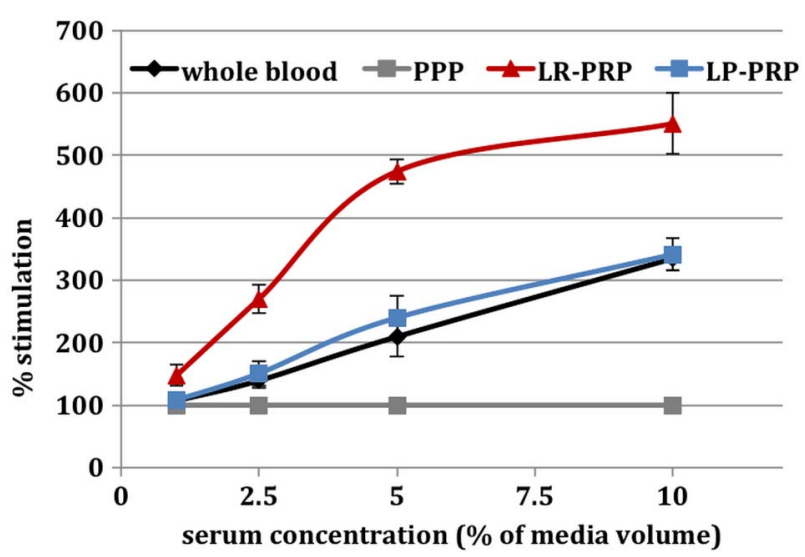

Figure 3 Stimulation of tendon cell proliferation. Data represent the mean $\pm S D$ of preparations from five individual donors. All preparations from each donor were assayed in parallel using reactivated platelet-poor plasma as the baseline control for each serum concentration tested.

The LP-PRP had a mean platelet increase of 2.2-fold over whole blood and mean platelet recoveries of $52 \%$ with a coefficient of variation of $19.4 \%$, indicating a high degree of variability. LP-PRP had WBCs reduced fivefold compared with whole blood. RBCs were reduced 100 -fold, to a haematocrit of 0.25 . Platelet function in the LP-PRP was deficient, as evidenced by a failure to consistently coagulate and undergo clot retraction when reactivated with $\mathrm{CaCl}_{2}$ alone. Consistent with this, serum from LP-PRP did not demonstrate elevated growth factor levels (figure 4), and the stimulation of tendon cell proliferation was not significantly different than serum from whole blood (figure 3). This deficiency in platelet activation was at least in part attributable to a failure of the LP-PRP to generate sufficient thrombin because addition of exogenous thrombin rescued the

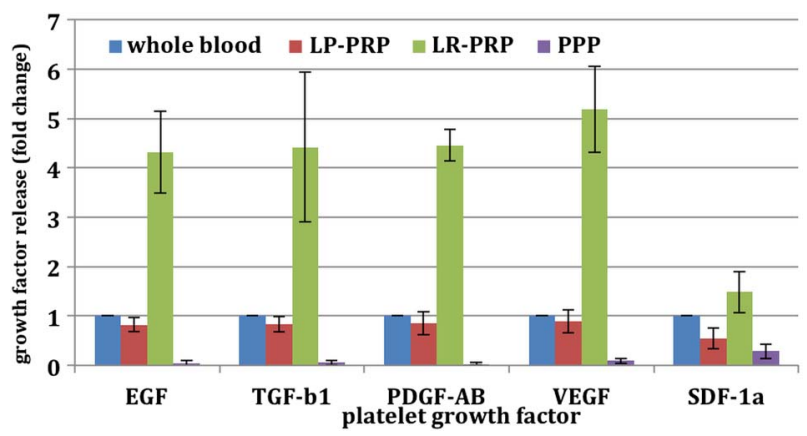

Figure 4 Serum growth factor release. Platelet-poor plasma, whole blood and platelet concentrates from the LP-PRP and LR-PRP systems were reactivated with a final concentration of $25 \mathrm{mM} \mathrm{CaCl}_{2}$ for $80 \mathrm{~min}$ at room temperature. Growth factor levels were measured in the resulting serum by ELISA. Bars represent the mean $\pm S D$ from five independent donors. All preparations from each donor were assayed in parallel. LP-PRP, leucocyte-poor platelet-rich plasma; LR-PRP, leucocyte-rich platelet-rich plasma.
Table 6 Serum growth factor levels $(\mathrm{pg} / \mathrm{mL})$ following reactivation with $\mathrm{CaCl}_{2}$

\begin{tabular}{lllll}
\hline $\begin{array}{l}\text { Platelet } \\
\text { growth } \\
\text { factor }\end{array}$ & $\begin{array}{l}\text { Whole } \\
\text { blood }\end{array}$ & $\begin{array}{l}\text { LP-PRP } \\
\text { system }\end{array}$ & $\begin{array}{l}\text { LR-PRP } \\
\text { system }\end{array}$ & PPP \\
\hline EGF & 2880 & 2350 & 12475 & 130 \\
TGF- $\beta 1$ & 4820 & 3908 & 19992 & 236 \\
PDGF-AB & 35580 & 29140 & 158480 & 1160 \\
VEGF & 269.6 & 264.4 & 1381.6 & 19.2 \\
SDF-1 $\alpha$ & 4975 & 4755 & 9530 & 3840 \\
\hline
\end{tabular}

EGF, epidermal growth factor; LP-PRP, leucocyte-poor platelet-rich plasma system; LR-PRP, leucocyte-rich platelet-rich plasma system; PDGF-AB, platelet-derived growth factor AB; PPP, platelet-poor plasma; SDF- $1 \alpha$, stromal-derived factor $1 \alpha$; TGF- $\beta 1$, transforming growth factor $\beta 1$; VEGF, vascular endothelial growth factor.

coagulation deficit and improved platelet growth factor release (figure 5).

Recently published studies have demonstrated that the intimate interactions between platelets and other blood cells are crucial for the physiological mechanisms to prevent infection and promote healing in the event of an injury, and that the failure of these mechanisms can promote pathological tissue states that contribute to disease. $^{8}{ }^{11}{ }^{17-25} 34-37$ The current study further contributes to this body of evidence, in that it uncovers a deficiency in platelet function with respect to growth factor release and the ability of the releasate to stimulate tendon cell proliferation when RBCs and leucocytes are lacking. A limitation of this study is that a detailed analysis of tendon cell viability was not conducted. Therefore, although the CellTiter-Glo assay that was used to determine cell proliferation measures the production of ATP specifically from viable cells, the relative contribution from prevention of apoptotic cell death and/or cellular senescence remains to be determined. In addition, it is well established that exposure of tendon fibroblasts to high levels of TGF- $\beta 1$ in vitro can activate their trans-differentiation to myofibroblasts $\left(\right.$ review by $\left.\mathrm{Hinz}^{38}\right)$. Myofibroblasts play an integral role in wound healing reactions due to their induced expression of smooth muscle actin that results in an acquired ability to contract and to contribute to physiological tissue repair. ${ }^{38}$ Myofibroblast contraction also leads to the liberation of TGF- $\beta 1$ from these cells, ${ }^{38}$ leading to the potential of a feed-forward mechanism to promote further fibroblast trans-differentiation. Further research will be required to determine the extent to which LR-PRP promotes fibroblast trans-differentiation, as this may represent an important mechanism through which PRP therapy may provide clinical benefit. Another important limitation of this study is that it is an in vitro study comparing two polar opposite platelet concentrate formulations (type 1a and type 3b). ${ }^{5}$ Therefore, the conclusions remain to be validated in animal models or the clinical setting. Further studies will be required to elucidate the precise individual contributions of platelets, 
Figure 5 Growth factor release from LP-PRP system preparations. Bars represent the mean $\pm S D$ of the fold change in serum growth factor content of LP-PRP activated with $\mathrm{CaCl}_{2}$ (blue bars; $n=5$ donors) or exogenously with $\mathrm{CaCl}_{2}+$ thrombin (red bars; $n=7$ donors). ${ }^{*} p<0.03$ compared with whole blood. ${ }^{* *} \mathrm{p}<0.01$ compared with whole blood. The green line represents whole blood platelet growth factor levels (set to 1-fold for comparison). LP-PRP, leucocyte-poor platelet-rich plasma.

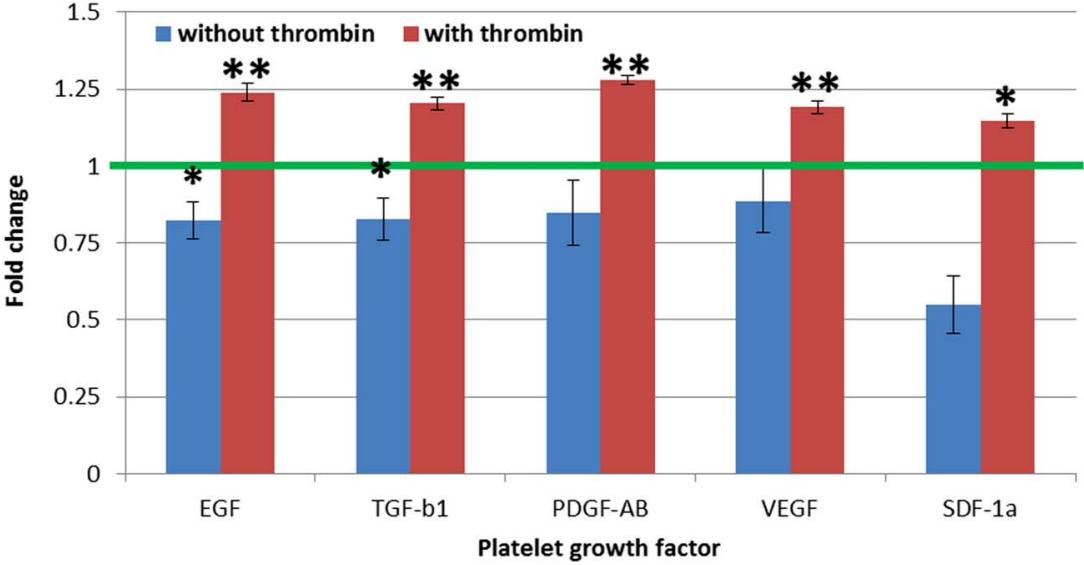

Platelet growth factor
RBCs and WBCs to the beneficial effects of PRP that are emerging in the scientific literature for various clinical applications. $^{3-5} 7$

\section{CONCLUSION}

LP-PRP did not demonstrate robust coagulation and platelet growth factor release compared with whole blood and LR-PRP. In addition, serum derived from LP-PRP did not provide a significant advantage to tendon cell proliferation compared with whole blood serum, although the LP-PRP contained a 2.2-fold increase in platelet concentration. These findings suggest that the non-platelet cellular components that are excluded from the LP-PRP are important for optimal platelet function, including thrombin generation leading to robust coagulation, growth factor release and the resulting capacity of the serum to stimulate cell proliferation. Future studies should be conducted to validate these findings in vivo, as these findings may have profound implications for how platelet concentrates are prepared and utilised in clinical applications.

Contributors WRP, BR, JH and BS conceived and designed the experiments. WRP analysed the data. WRP and BR wrote the first draft of the manuscript. WRP, MM and SB contributed to the writing of the manuscript. WRP, BR, JH, $\mathrm{SB}, \mathrm{MM}$ and $\mathrm{BS}$ agree with manuscript results and conclusions. WRP, BR, SB and MM jointly developed the structure and arguments for the paper. WRP, $\mathrm{BR}, \mathrm{JH}, \mathrm{BS}, \mathrm{MM}$ and $\mathrm{SB}$ made critical revisions and approved final version. All authors reviewed and approved of the final manuscript.

Funding Support for this research was provided by DePuy Synthes Mitek Sports Medicine, Raynham, Massachusetts, USA.

Competing interests WRP, BR, JH, SB and BS are employees of DePuy Synthes Mitek Sports Medicine, a Johnson \& Johnson Company. MM is a paid consultant for DePuy Synthes Mitek Sports Medicine and an employee of Health Economics Associates, LLC.

Provenance and peer review Not commissioned; externally peer reviewed.

Open Access This is an Open Access article distributed in accordance with the Creative Commons Attribution Non Commercial (CC BY-NC 4.0) license, which permits others to distribute, remix, adapt, build upon this work noncommercially, and license their derivative works on different terms, provided the original work is properly cited and the use is non-commercial. See: http:// creativecommons.org/licenses/by-nc/4.0/

\section{REFERENCES}

1. Marx RE. Platelet-rich plasma: evidence to support its use. J Oral Maxillofac Surg 2004;62:489-96.

2. Anitua E, Andia I, Ardanza B, et al. Autologous platelets as a source of proteins for healing and tissue regeneration. Thromb Haemost 2004;91:4-15.

3. Hall MP, Band PA, Meislin RJ, et al. Platelet-rich plasma: current concepts and application in sports medicine. J Am Acad Orthop Surg 2009;17:602-8.

4. Sampson S, Gerhardt M, Mandelbaum B. Platelet rich plasma injection grafts for musculoskeletal injuries: a review. Curr Rev Musculoskelet Med 2008;1:165-74.

5. Mishra A, Harmon K, Woodall J, et al. Sports medicine applications of platelet rich plasma. Curr Pharm Biotechnol 2012;13:1185-95.

6. Ahmad Z, Howard D, Brooks RA, et al. The role of platelet rich plasma in musculoskeletal science. JRSM Short Rep 2012;3:40.

7. Halpern BC, Chaudhury S, Rodeo SA. The role of platelet-rich plasma in inducing musculoskeletal tissue healing. HSS J 2012;8:137-45.

8. Bielecki T, Dohan Ehrenfest DM, Everts PA, et al. The role of leukocytes from L-PRP/L-PRF in wound healing and immune defense: new perspectives. Curr Pharm Biotechnol 2012;13:1153-62.

9. Chang KV, Hung CY, Aliwarga F, et al. Comparative effectiveness of platelet-rich plasma injections for treating knee joint cartilage degenerative pathology: a systematic review and meta-analysis. Arch Phys Med Rehabil 2014;95:562-75.

10. Dohan Ehrenfest DM, Bielecki T, Jimbo R, et al. Do the fibrin architecture and leukocyte content influence the growth factor release of platelet concentrates? An evidence-based answer comparing a pure platelet-rich plasma (P-PRP) gel and a leukocyteand platelet-rich fibrin (L-PRF). Curr Pharm Biotechnol 2012;13:1145-52.

11. Elstad MR, Mclntyre TM, Prescott SM, et al. The interaction of leukocytes with platelets in blood coagulation. Curr Opin Hematol 1995:2:47-54.

12. Santos MT, Valles J, Aznar J, et al. Role of red blood cells in the early stages of platelet activation by collagen. Thromb Haemost 1986;56:376-81.

13. Riboh JC, Saltzman BM, Yanke AB, et al. Effect of leukocyte concentration on the efficacy of platelet-rich plasma in the treatment of knee osteoarthritis. Am J Sports Med 2015. doi:10.1177/ 0363546515580787

14. McCarrel TM, Minas T, Fortier LA. Optimization of leukocyte concentration in platelet-rich plasma for the treatment of tendinopathy. J Bone Joint Surg Am 2012;94:e143-8.

15. Braun $\mathrm{HJ}$, Kim HJ, Chu CR, et al. The effect of platelet-rich plasma formulations and blood products on human synoviocytes: implications for intra-articular injury and therapy. Am J Sports Med 2014;42:1204-10. 
16. Dragoo JL, Braun HJ, Durham JL, et al. Comparison of the acute inflammatory response of two commercial platelet-rich plasma systems in healthy rabbit tendons. Am J Sports Med 2012;40:1274-81.

17. Semple JW, Italiano JE Jr, Freedman J. Platelets and the immune continuum. Nat Rev Immunol 2011;11:264-74.

18. Valles J, Santos MT, Aznar J, et al. Erythrocytes metabolically enhance collagen-induced platelet responsiveness via increased thromboxane production, adenosine diphosphate release, and recruitment. Blood 1991;78:154-62.

19. Valles J, Santos MT, Aznar J, et al. Platelet-erythrocyte interactions enhance alpha(Ilb)beta(3) integrin receptor activation and P-selectin expression during platelet recruitment: down-regulation by aspirin ex vivo. Blood 2002;99:3978-84.

20. Marcus AJ, Safier LB. Thromboregulation: multicellular modulation of platelet reactivity in hemostasis and thrombosis. FASEB $J$ 1993;7:516-22.

21. Broos K, Feys HB, De Meyer SF, et al. Platelets at work in primary hemostasis. Blood Rev 2011;25:155-67.

22. Cerletti C, de Gaetano G, Lorenzet R. Platelet-leukocyte interactions: multiple links between inflammation, blood coagulation and vascular risk. Mediterr J Hematol Infect Dis 2010;2: e2010023

23. Del Maschio A, Dejana E, Bazzoni G. Bidirectional modulation of platelet and polymorphonuclear leukocyte activities. Ann Hematol 1993;67:23-31.

24. Ghasemzadeh M, Hosseini E. Platelet-leukocyte crosstalk: linking proinflammatory responses to procoagulant state. Thromb Res 2013;131:191-7.

25. Jenne CN, Urrutia R, Kubes P. Platelets: bridging hemostasis, inflammation, and immunity. Int J Lab Hematol 2013;35:254-61.
26. Versteeg $\mathrm{HH}$, Heemskerk JW, Levi M, et al. New fundamentals in hemostasis. Physiol Rev 2013;93:327-58.

27. Weyrich AS, Zimmerman GA. Platelets: signaling cells in the immune continuum. Trends Immunol 2004;25:489-95.

28. Zarbock A, Polanowska-Grabowska RK, Ley K. Platelet-neutrophil-interactions: linking hemostasis and inflammation. Blood Rev 2007;21:99-111.

29. Enoch S, Leaper DJ. Basic science of wound healing. Surgery (Oxford) 2005;23:37-42.

30. McClatchey AI, Yap AS. Contact inhibition (of proliferation) redux. Curr Opin Cell Biol 2012;24:685-94.

31. de Witt SM, Verdoold R, Cosemans JM, et al. Insights into platelet-based control of coagulation. Thromb Res 2014;133:S139-48

32. Boswell SG, Cole BJ, Sundman EA, et al. Platelet-rich plasma: a milieu of bioactive factors. Arthroscopy 2012;28:429-39.

33. Boswell SG, Schnabel LV, Mohammed HO, et al. Increasing platelet concentrations in leukocyte-reduced platelet-rich plasma decrease collagen gene synthesis in tendons. Am J Sports Med. 2014;42:42-9.

34. Blair P, Flaumenhaft R. Platelet alpha-granules: basic biology and clinical correlates. Blood Rev 2009;23:177-89.

35. Cerletti C, Evangelista V, De Gaetano G. Polymorphonuclear leucocyte-dependent modulation of platelet function: relevance to the pathogenesis of thrombosis. Pharmacol Res 1992;26:261-8.

36. Marcus AJ. Neutrophils inhibit platelet reactivity by multiple mechanisms: relevance to thromboregulation. J Lab Clin Med 1990;116:138-9.

37. Rendu F, Brohard-Bohn B. The platelet release reaction: granules' constituents, secretion and functions. Platelets 2001;12:261-73.

38. Hinz B. Formation and function of the myofibroblast during tissue repair. J Invest Dermatol 2007;127:526-37. 